\title{
TRATAMIENTO DE UN CASO DE HIPOCONDRÍA CON RITUALES OBSESIVOS
}

\author{
Josep A. Pena I Garijo \\ Psicólogo Clínico
}

\begin{abstract}
RESUMEN
Se relata la intervención realizada en un caso de trastomo hipocondriaco en el que la paciente, una mujer joven, presenta una preocupación morbose por la posibilidad de padecer un cancer de mama y/o genital. Esta preocupación le provocaba una considerable ansiedad, la cual reducla a través de un comportamiento compulsivo de auto-exploración mamaria, que llegó a ocasionarle problemas físicos en los senos. Se ejemplifica la aplicación de un programa multicomponente en el que se combrnaron estrategias de exposición con prevención de respuesta, habit reversal y técntcas de reducción de ansiedad, asi como un componente cognitivo que se desarrollo a lo largo de todas las sesiones. Se expone también la dificultad que sobreviene en ocasiones para establecer el diagnóstico diferencial entre la hipocondrla y el trastomo obsesivo-compulsivo.
\end{abstract}

Palabras clave: Estudio de caso; hipocondrla; trastorno obsesivo-compulsivo; it tuales; exposición con prevención de respuesta.

\begin{abstract}
The psychological intervention in a case of hypochondriasis with an obsessive component is presented. The patient was a young female with a morbid preoccupation about the possibility of having a breast and/or genital cancer. This preoccupation was making her considerabily anxious, and she tried to reduced such anxiety by compulsively checking her breasts, wich resulted in physical problems. We show the application of a multicomponent program in which exposure strategies plus response prevention, habit reversal and anxiety reduction techniques were combined. A cognitive component developed through the sessions of treatment, as well. Finally, the dificulty of making a differential diagnosis between hypochondriasis and obsessive-compulsive disorder is also adressed.
\end{abstract}

Key words: Case study; hypochondriasis; obsessive-compulsive disorder; rituals; exposure with response prevention.

Correspondencia: Unidad de Salud Mental "Castello II". Servei Valencià de Salut. Hospital Provincial de Castello. Av. del Dr. Clará 19. 12004 Castelló de la Plana. Teléfono 964-359771 


\section{INTRODUCCIÓN}

La hipocondría se describe en el DSM IV (APA, 1995) como preocupación por el miedo a tener, o la convicción de padecer, una enfermedad grave a partir de la interpretación personal de síntomas somáticos (Criterio A). El Criterio B se refiere a que la preocupación persiste a pesar de las exploraciones y explicaciones médicas apropiadas.

A pesar de ser éste un desorden conocido hace cientos de años, su consideración como un trastorno de muy dificil tratamiento ha sido la idea predominante hasta la actualidad. En los últimos 30 años el abordaje de la conducta anormal de enfermedad ha resurgido poderosamente desde la óptica cognitivo-conductual, motivado en parte por el éxito terapéutico obtenido desde este modelo en otros trastornos psicológicos y coincidiendo, sin duda, con la necesidad que plantea el tratamiento de un tipo de psicopatologia que ocasiona una considerable repercusión sobre los costos (económicos $y$ en otros recursos) y en la dinámica de los servicios de asistencia sanitaria (Martinez y Botella, 1996).

Precisamente, desde un servicio de salud público se plantea la intervención que relatamos a continuación y de la que quisiéramos resaltar la brevedad en tiempo y número de sesiones, con to que ello supone de alivio relativo a la presión asistencial a la que normalmente nos vemos sometidos en este contexto de atención sanitaria.

En el caso que nos ocupa, se cumplen los criterios a los que aludiamos al principio. También el diagnóstico diferencial con el trastorno delirante, el trastorno dismórfico corporal y los trastornos de ansiedad. Sin embargo, como veremos en la presentación clínica, la distinción con respecto al trastorno obsesivo-compulsivo no resulta tan sencilla. En ambos supuestos se da un componente fóbico: el miedo a la enfermedad, y también en ambos casos se dan conductas de evitación (o rituales conjugatorios). El modelo conductual del miedo postula que el mantenimiento de éste se produce por la reducción de la activación mediante el reforzamiento negativo de la conducta de evitación. En el trastorno obsesivo, el papel reductor de la ansiedad que produce la idea obsesiva ( $p$. ej., el miedo a contaminarse), 10 cumple el ritual compulsivo (lavarse). En el caso de la hipocon- dria, sobre todo cuando el componente predominante es el miedo a la enfermedad o el dolor, la reducción de la activación asociada a ese miedo, se produce al ejecutar algún componente "ritual" como ir al médico, solicitar información sobre la supuesta enfermedad 0 , como en el sujeto que nos ocupa, la auto-exploración con fines tranquilizadores. Este fue el motivo principal por el que abordamos este caso con una técnica, la exposición con prevención de respuesta, que es precisamente la intervención "estrella" en los trastornos obsesivo-compulsivos.

En cualquier caso, y aunque el diagnóstico psiquiátrico es necesario (incluso imprescindible en determinados ámbitos), lo que debe, a nuestro juicio, dirigir fundamentalmente la intervención, son los resultados del análisis funcional de las conductas desadaptadas que llevan al paciente a la consulta y que, en último caso, se llamen como se llamen, producen un malestar significativo en nuestro paciente y han de ser abordadas mediante las estrategias de que dispongamos y sepamos resultan eficaces para su problema concreto.

\section{MÉTODO}

\subsection{Sujeto}

"C" es una mujer de 32 años de edad, casada $y$ con dos hijos pequeños que vive en una pequena población cercana a nuestra ciudad. Tiene estudios primarios y es operaria de una fábrica.

Acude a nuestro servicio remitida por el médico de cabecera que habia pautado Fluvoxamina $50 \mathrm{mg}$./dia, y con un diagnóstico de sospecha de obsesiones hipocondriacas.

Nacida a término, deambula y habla a la edad normal. El resto de su desarrollo psicomotor y el del control de esfínteres es evolutivamente adecuado. No recuerda enfermedades graves en la infancia y se describe como una nina normal, mala estudiante, pero bien adaptada a la vida social y familiar. Conoce a su marido a los 16 ańos y se casa a los 22 . Tiene dos partos normales. No padece alergias $u$ otras enfermedades conocidas y no tiene antecedentes psicológicos o psiquiátricos (exceptuando el tratamiento del médico de cabecera).

Relata que desde hace algunos meses tiene "obsesión" por la salud de su marido e hijos, pensando que les puede ocurrir algo grave, exagerando una simple caida o un resfriado. 
A raiz de la muerte de una chica del pueblo, a consecuencia de un cáncer de mama, comienza a tocarse los pechos y los genitales "buscando algún bulto o quiste". Piensa que ella "también va a tener un cáncer".

Preguntada por los antecedentes en la familia de enfermedades o trastornos psicológicos no recuerda ningún dato de interés. Sin embargo, su marido nos contará posteriormente que su madre es (textualmente) "una hipocondríaca".

\section{2.- Evaluación}

En este apartado incluimos la entrevista con la paciente, la evaluación mediante autoinformes y autorregistros, los resultados de una entrevista estructurada y la hipotesis sobre el problema. Para el diagnóstico psiquiátrico nos regimos por el Manual Diagnóstico y Estadístico de los Trastornos Mentales IV Edición (APA, 1995).

\subsection{1.- Entrevista con la paciente}

En el momento de la primera entrevista $C$ se encuentra eutimica, indica que no toma la medicación, y mantiene un nivel de ansiedad general leve.

Describe su problema como "siempre tengo que estar tocándome los pechos y mirándome bajo". Piensa que va a tener un cáncer "como la chica del pueblo". Cuando el médico la tranquiliza sobre sus síntomas, está mejor durante unos dias, tras los cuales, reaparece la preocupación.

Exagera cualquier sintoma de los hijos y está muy encima de ellos. Se preocupa si llegan tarde o no sabe donde están.

Se realiza una entrevista de screening y se plantea el diagnostico diferencial con el trastorno de ansiedad generalizada y el trastorno obsesivo-compulsivo. No presenta alteraciones del estado de ánimo, la percepción, el juicio o el curso del pensamiento. En cuanto al contenido del pensamiento, $C$ presenta una preocupación excesiva por la posibilidad de tener un cáncer (de mama o genital). Estas ideas no tienen en ningún momento carácter delirante. Realiza conductas compulsivas de auto-exploración mamaria con la finalidad de encontrar algún bulto $u$ otra senal que le "confirme el diagnóstico". La frecuencia $\theta$ intensidad de esta conducta ha llegado a producirle hematomas e irritación, lo cual, a su vez, aumenta la preocupación y le hace tocarse con más frecuencia.

No hay alteraciones del sueño o el apetito. Presenta una leve ansiedad flotante $y$, ocasionalmente, siente nauseas por la manana. EI aumento de ansiedad coincide con pequeñas preocupaciones cotidianas y con la exposición a estimulos que le recuerdan enfermedades (peliculas, conversaciones con vecinas, visitas al médico con los ninos, hospitales, etc.). Ejecuta algunos rituales de comprobación (puertas, llaves y gas), de intensidad subclinica.

La entrevista con el marido confirma lo expuesto por la paciente. Adopta una actitud tolerante con el problema de C y se muestra dispuesto a colaborar. Nos cuenta también, como hemos comentado antes, que la madre de la paciente está continuamente quejándose y verbalizando que tiene tal o cual enfermedad: "es muy aficionada a ir al médico". Confirma también que C se preocupa en exceso por los problemas más nimios y que, últimamente, está más nerviosa. La vida de relación, la conducta sexual y el nivel de actividad son buenos.

\subsection{2.- Entrovista estructurada, autoinformes y autoobservación}

En primer lugar se le aplico:

- Entrevista Diagnóstica Estructurada para el diagnóstico de hipocondria (Barsky y col., 1992).

Se solicito de la paciente que cumplimentara los siguientes cuestionarios:

- EAE. Escala de Actitudes ante la Enfermedad (Kellner, 1986).

- Inventario de Depresión de Beck (1983).

- OCM. Inventario obsesivo compulsivo del Maudsley (Hodgson y Rachman, 1977).

- STAI. Inventario de Ansiedad Estado-Rasgo (Spielberger, 1977). Sólo se aplicó la parte B, correspondiente a la ansiedad-rasgo.

Al finalizar la sesión se le entregó a la paciente un registro de autoobservación que elaboramos ad hoc donde debla anotar las incidencias relacionadas con su problema, especialmente los pensamientos sobre enfermedades y las conductas de autoexploración. (Tabla 1).

\subsection{3.- Hipótesis explicativa}

Los autorregistros mostraron claramente una relación entre determinados estímulos y la 
aparición de las ideas hipocondriacas, definidas éstas como la preocupación por padecer un cáncer de mama y/o genital o, en su caso, otra enfermedad grave.

\section{Tabla 1.- Autorregistro}

\begin{tabular}{|l|l|l|l|l|}
\hline $\begin{array}{l}\text { DWa } \\
\text { Hora }\end{array}$ & $\begin{array}{l}\text { Stuación } \\
\begin{array}{l}\text { ¿Qué } \\
\text { estaba } \\
\text { pasando? }\end{array}\end{array}$ & $\begin{array}{l}\text { Emocion } \\
\text { ¿Como } \\
\text { me } \\
\text { siento? }\end{array}$ & $\begin{array}{l}\text { Peneamiento } \\
\text { ¿Qué } \\
\text { estoy } \\
\text { pensando? }\end{array}$ & $\begin{array}{l}\text { Conduct } \\
\text { ¿Qué } \\
\text { hago? }\end{array}$ \\
\hline & & & & \\
& & & \\
\hline
\end{tabular}

Para realizar el análisis funcional de esta paciente nos basamos en la propuesta de Avia (Ver Avia, 1993. pag. 124). Ponemos en negrita los componentes hipocondriacos en los tres sistemas de respuesta:

1.- Los antecedentes remotos parecen estar centrados en el posible aprendizaje por modelado de la madre (según el marido, "una hipocondriaca").

2.- C presentaba una preocupación importante por la posibilidad de padecer un cáncer de mama. Los antecedentes próximos de esta preocupación parecen encontrarse en la muerte de una vecina de su pueblo a causa de esta enfermedad. A partir de este momento empiezan a aparecer pensamientos relacionados con el tema (respuesta cognitiva). Estos la motivan a consultar a su médico acerca de la posibilidad de estar desarrollando un tumor (respuesta motora). El médico la tranquiliza (reduce su ansiedad). Esta momentánea tranquilidad actúa como refuerzo de la conducta de visitar al médico (lo cual se hace frecuente) y, además, se generaliza a otras conductas tendentes a reducir la ansiedad, como auto-explorarse (respuesta motora). En este momento la secuencia conductual sería como sigue:

Estímulo (oir hablar de enfermedades, peliculas o noticias relacionadas, los hospitales o centros de salud)__Respuesta cognitiva ("creo que tengo un tumor", "tendré un cáncer", etc.)-Respuesta fisiológica (aumento activación)Respuesta motora (tocarse los pechos con la finalidad de encontrar un bulto)-Consecuencia (reducción momentánea de la ansiedad, que actúa como refuerzo negativo).

Esta primera secuencia, bastante simple, se complica cuando $\mathrm{C}$ empieza a interpretar pequeños cambios en su organismo (respuesta cognitiva), cambios que las personas "normales" apenas percibimos o no les prestamos atención, como indicativo del posible padecimiento. Esto es coherente con las hipótesis actuales sobre la hipocondria que apuntan hacia la hipervigilancia de las propias sensaciones corporales como un factor importante en el desarrollo y mantenimiento de la enfermedad (Easterning y Leventhal, 1989; Warwick y Salkovskis, 1990; Hitchcock y Mathews, 1992; Avia, 1993). Además, la continua manipulación de sus senos le llegó a producir una importante irritación y la aparición de hematomas en esta zona, lo cual servia por sí mismo como estímulo desencadenante de las cogniciones hipocondriacas y la posterior conducta de exploración.

\section{3.- Tratamiento}

Después de realizar el análisis funcional y formular la hipótesis explicativa del problema, elaboramos, junto con la paciente, una serie de objetivos y establecimos los criterios de cumplimiento de los mismos (el autor de estas lineas tiene como costumbre preguntar al paciente "¿qué es exactamente lo que pretendes conseguir viniendo aqui?" y le pide que lo anote en una hoja con la finalidad de establecer un criterio de satisfacción subjetiva con la intervención).

\subsection{1.- Objetivos de la Intervención y criterio de consecución}

1.- Reducción del nivel general de ansiedad. La paciente debería evaluar su ansiedad general por debajo de "2" (se enseño a la paciente a medir su ansiedad en USAs, pero en una escala de 0 a 10, ya que hemos comprobado en la práctica que resulta más sencillo para los pacientes evaluarlo de esta manera). 
2.- Desaparición de los rituales de autoexploración mamaria. La paciente debería ser capaz de no tocarse los pechos, excepto una vez al mes, después de la menstruación. En ese momento se le permitia la auto-exploración con fines preventivos.

3.- Detección y control de los pensamientos hipocondriacos (tener un cáncer u otra enfermedad grave). El marido deberia informar de la frecuencia de las quejas anotando éstas en un diario. También debería disminuir la frecuencia de pensamientos en el autorregistro hasta un límite que juzgáramos subjetivamente razonable (de acuerdo con la paciente). Este punto resulta polémico ya que una preocupación razonable por la propia salud es absolutamente adaptativa y su ausencia, peligrosa. Así mismo, existen circunstancias normales, como el embarazo (Fava, Grandi, Saviotti, Trombini y Orlandi, 1990), o condiciones de salud alterada en las que la preocupación por llevar una dieta adecuada, o dejar de fumar, o consultar al médico ante un síntoma "real", u otras manifestaciones de esa preocupación, son conductas perfectamente sanas.

4.- Desaparición de los rituales de comprobación. Sólo deberia comprobar el gas, las puertas y la llave una vez.

5.- Deberla poder hablar con las vecinas, contemplar un programa de televisión o leer una revista sobre enfermedades sin experimentar ansiedad.

6.- Resistir el impulso de ir al médico excepto cuando se tratara de un sintoma no relacionado con el cáncer de mama (no se contempló la preocupación por el cáncer genital ya que pensamos que desapareceria con la simple información del médico y con la pauta de exploraciones programadas del ginecólogo).

\subsection{2.- Planificación de la intervención}

Para la consecución de los objetivos propuestos se escogieron las siguientes estrategias:

- Para el objetivo número 1: Entrenamiento en relajación progresiva. Se utilizó la versión de Bernstein y Borkovec (1973).

- Para los objetivos número 2 y 4: Exposición con prevención de respuesta.

- Para el objetivo número 3: Información sobre su problema en términos conductuales. Utiliza- ción del autorregistro (ver tabla número 1). Parada de pensamiento (Bellack y Hersen, 1977). Reestructuración cognitiva mediante reatribución y confrontación con la realidad.

- Para el objetivo número 5: Exposición in vivo a las situaciones que le provocaban ansiedad o disparaban los pensamientos sobre enfermedades.

- Para el último objetivo, hablamos con el médico de cabecera y el ginecólogo instruyéndoles en el manejo de esta paciente: explicación clara de sus síntomas con visitas de revisión programadas. También se explicó a la paciente que no debería consultar a demanda, excepto en los casos previstos en que pudiera aparecer otra sintomatologia distinta de la objeto de modificación.

Incluimos a $\mathrm{C}$ en un grupo de relajación breve, que dirige la enfermera del centro de salud, para recibir entrenamiento en esta técnica. Se desarrolló paralelamente a la intervención individual durante las 4 primeras semanas (una sesión semanal).

La intervención se prolongó durante tres meses con un total de 7 sesiones en las que se fue desarrollando el programa previsto.

\subsubsection{Desarrollo de la intervención sesión a sesión}

En la primera sesión, después de la de evaluación se llevó a cabo la explicación del trastorno de la paciente en términos conductuales. Se la tranquilizo sobre sus sintomas, partiendo de que su preocupación y su ansiedad eran "reales", pero atribuyéndolos poco a poco a procesos psicológicos y no a causas orgánicas. Utilizamos un modelo explicativo similar al del pánico (Beck, 1988; Clark, 1987; Salkovskis y Clark, 1993). También se la instruyó en la técnica de exposición in vivo. Tras explicarle sus fundamentos teoricos, se le pidió que permaneciera delante del espejo de su habitación (donde solia realizar sus "toqueteos") durante dos horas en dias altemos y con la condición de "no tocarse" durante ese tiempo. Deberia también registrar en un diario, que le dimos al efecto, la intensidad de la ansiedad al inicio y al final de cada sesión de auto-exposición. Para los rituales de comprobación deberia tan sólo evitar hacerlo, para lo cual se le sugirió que cada vez que apagara el gas, cerrara la 
puerta o metiera las llaves en el bolso, se dijera a si misma "ya está hecho, no debo comprobarto de nuevo". En caso de aparecer la tendencia a comprobar, sencillamente debería aguantar la ansiedad mediante un sencillo ejercicio de respiración y esperar a que descendiese.

En la segunda sesión se observó que tenia dificultades para prevenir la respuesta de tocarse los pechos durante la tarea de exposición. Por lo que decidimos incluir un elemento de habit reversal: se pidió a la paciente que identificara el momento en que "se le disparaba la mano" y se le sugirió que realizara un movimiento similar (mismo gesto, mismos grupos musculares) pero con un "objetivo diferente"; en este caso se palpaba las dos manos entre sí. También modelamos en esta sesión la detención de pensamiento. Realizamos el entrenamiento con la palabra "PARA" en voz alta y la lectura posterior de una tarjeta que elaboramos al efecto con un contenido agradable (descripción de su hijo jugando con ella en el parque). Se le pidió que elaborara tres tarjetas más para la siguiente sesión y se le pidió que repitiera este ejercicio en casa durante tres días, tras los cuales debla intentar hacerlo subvocalmente. Se le indicó que lo utilizara siempre que le viniera al pensamiento la idea de padecer un cáncer o de tocarse los pechos. Acabamos la sesión profundizando en el modelo explicativo que le habiamos propuesto y elaborando a la luz de las situaciones del auto-registro una interpretación más acorde de sus sintomas.

En las siguientes sesiones ( 3 a 5) continuamos con la reestructuración de sus creencias hipocondriacas aprovechando las situaciones que iban apareciendo en los registros.

Entre la sesión 3 y 4 programamos una cita en la Asociación Española Contra el Cáncer (que además está frente a nuestro hospital) para que hablara con un grupo de gente y visionara un video educativo sobre el cáncer de mama.

Entre las sesiones 4 y 5 debia realizarse una exploración programada de sus senos, la cual realizó sin experimentar ansiedad.

Tras la sesión 5, repasamos las estrategias de afrontamiento y pautamos la continuación de las tareas de exposición (una vez a la semana). Se volvieron a pasar el OCM, el BDI, el STAI y el EAE, asi como la Entrevista de Barsky.

Se programaron dos seguimientos a los tres y seis meses. El último seguimiento se realizó por teléfono hace algunos dias.

\section{3.- RESULTADOS}

En la evaluación pre-tratamiento los cuestionarios arrojaron los siguientes datos (Tabla 2):

Tabla 2.- Resultado de las evaluaciones pre y post-tratamiento

\begin{tabular}{|l|c|c|}
\hline & PRE & POST \\
\hline $\begin{array}{l}\text { BDI (Inventario de } \\
\text { Depresión de Beck) }\end{array}$ & 3 & 3 \\
$\begin{array}{l}\text { EAE (Escala de Actitudes } \\
\text { hacia la enfermedad) } \\
\text { OCM (Inventario Obsesivo- } \\
\text { Compulsivo del Maudsley) } \\
\text { STAI (Ansiedad Rasgo) }\end{array}$ & 13 & 16 \\
\hline
\end{tabular}

El BDI confirmó lo observado durante la entrevista (P.D.=6; depresión ausente). EI OCM da una puntuación global de 13. Los items positivos son los relacionados con conductas rituales de comprobación, miedo a las enfermedades y miedo a contaminarse. Cuando se le preguntó en la sesión siguiente acerca de sus respuestas al cuestionario, aclaró que el miedo que tenia no era a contaminarse o infectarse sino a "coger una enfermedad". No efectúa rituales de limpieza y los de comprobación son leves.

EI EAE arrojó una puntuación global de 62, indicativo de una hipocondria severa. Los subfactores se presentan en la Tabla 3 (puntuaciones directas).

Tabla 3.- Escalas del EAE

\begin{tabular}{|l|c|c|}
\hline Escala & Pre & Post \\
\hline Preocupación por la & & \\
enfermedad & 12 & 4 \\
Preocupación por el dolor & 10 & 2 \\
Habitos de salud & 8 & 4 \\
Creencias hipocondriacas & 8 & 0 \\
Tanatofobia & 8 & 2 \\
Fobia a la enfermedad & 4 & 0 \\
Preocupación por el cuerpo & 12 & 2 \\
Experiencia con el tratamiento & 4 & 2 \\
Efectos de ios sintomas & 0 & 0 \\
\hline
\end{tabular}


En el STAl presentaba una ansiedad-rasgo moderadamente elevada (PC: 60). Por este motivo incluimos en la intervención una estrategia de reducción de la ansiedad, concretamente la técnica de la relajación progresiva (Berstein y Borkovec, 1973).

La entrevista estructurada de Barsky (1992) permitió confirmar el diagnóstico de hipocondria siguiendo los criterios del DSM IV (la entrevista se basa en los criterios DSM III-R; en este caso hemos asumido la equivalencia).

En la evaluación post-tratamiento se aprecia que, excepto el BDI que no se modificó (nunca estuvo alterado), los demás autoinformes sugieren que los objetivos previstos se hablan cumplido. Las puntuaciones del EAE habian sufrido un considerable descenso (P.D.: 16), asi como las del OCM (P.D.: 5) y del STAI (P.C.: 35) y, lo más importante, ya no cumplia los criterios de hipocondria del DSMIV, demostrado a través de la entrevista de Barsky.

Los rituales de comprobación hablan cedido desde el principio y la preocupación por padecer un cáncer genital, como habiamos supuesto, desapareció sin intervenir (bastó la explicación del ginecólogo y la programación de visitas semestrales). En la cita que programamos en la Asociación Contra el Cáncer, nos sorprendió gratamente el hecho de que no experimentó apenas ansiedad y que, además, salio muy aliviada al darse cuenta que era capaz de pensar que "ella ya no tenia esos sintomas". De todos modos, le pedimos que grabara algunos programas de televisión del tipo "Más vale prevenir" y los visionara durante dos horas una vez a la semana, asi como que no evitara estos programas si le aparecian casualmente. Si experimentaba ansiedad debería aplicar la relajación en su formato breve. La única condición que impusimos fue que no dejara de vertos hasta que no le desapareciera la posible ansiedad. No hubo problemas al respecto. Las conversaciones con las vecinas sobre estos temas dejaron de producirle malestar, e incluso llegó a comentarnos que "eran unas hipocondriacas".

En el primer seguimiento al mes, continuaba asintomática, aunque estaba preocupada por problemas laborales. Continuaba utilizando la re-

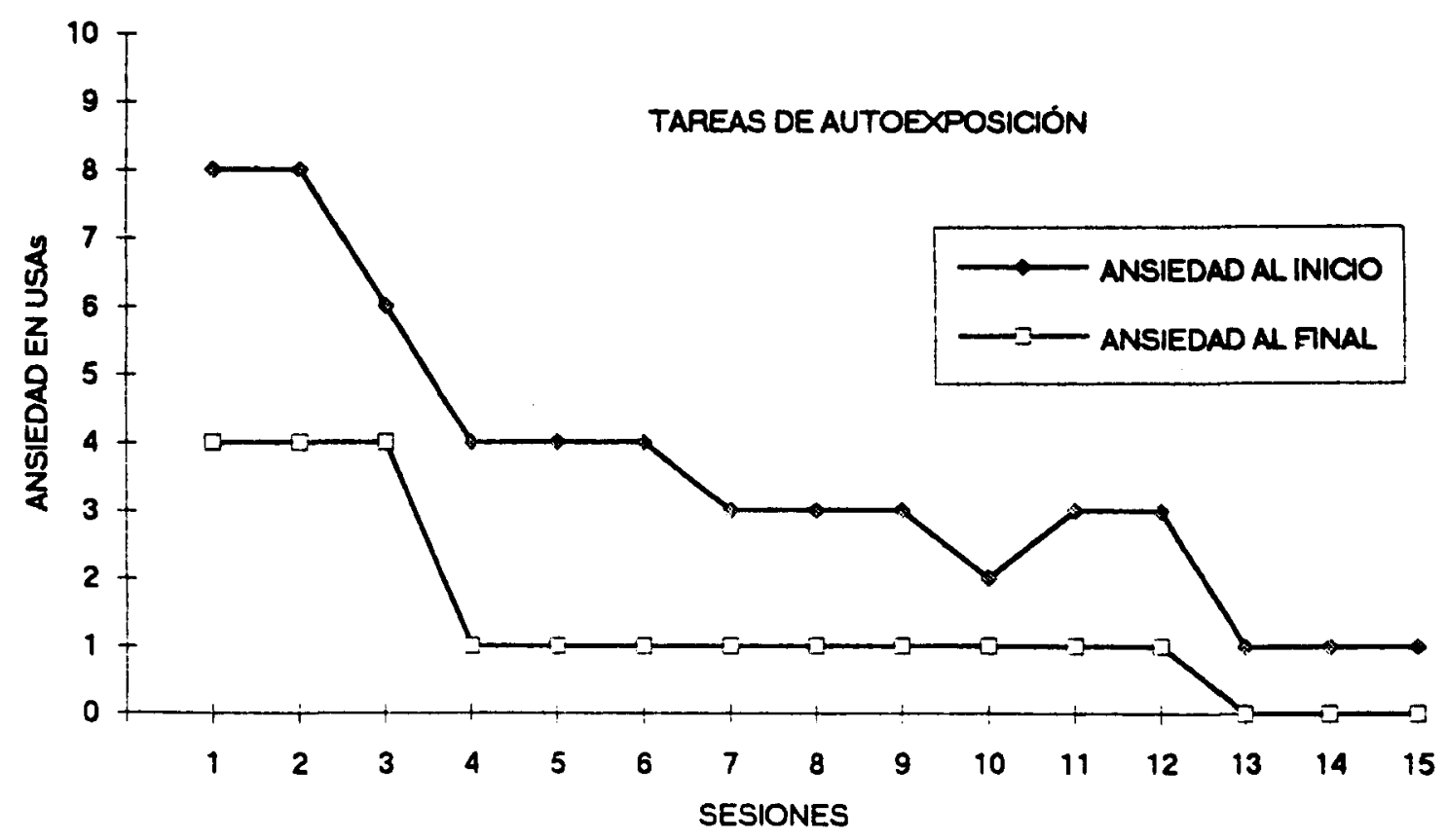

Figura 1.- 
lajación y se exponía tal y como habiamos previsto. Habia realizado otra auto-exploración después de la menstruación sin experimentar ansiedad alguna. Los registros no mostraban ninguna alteración importante: habia hecho tres anotaciones en todo el mes coincidiendo con la noticia de una enfermedad de un familiar, que habla afrontado adecuadamente. Su marido confirmó la mejoría y nos explicó que "ya no daba tanto la lata a sus hijos".

Entre este primer seguimiento y el segundo que se habia programado, $\mathrm{C}$ volvió a consultar por encontrarse nerviosa y preocupada. Estos sintomas coincidian con haber tenido que dejar de trabajar y tener que acompañar a su suegro al IVO (Instituto Valenciano de Oncologia). No habian reaparecido los rituales, pero estaba disgustada y tenía miedo de recaer. Se la tranquilizo al respecto y se repasaron las estrategias que habia aprendido durante la intervención. Un mes después se encontraba bien.

La ansiedad, como se ponia de manifiesto a través de los registros de auto-exposición, habia ido descendiendo, más al principio, y se mantenia por debajo de la puntuación "2" (Figura 1). Del mismo modo, la frecuencia de aparición de las ideas hipocondriacas se encontraba muy disminuida, excepto el cuarto mes en que se observa, a través de los autorregistros, una leve subida a consecuencia de la noticia de la enfermedad de su suegro.

Es interesante observar el espectacular descenso en la frecuencia de los rituales de autoexploración mamaria (Figura 2). La mera instrucción de intentar no tocarse los pechos habia tenido un efecto de reducción de la frecuencia de 8610 al dia cuando llegó a consulta, a tan sólo 5 durante esa primera semana de registro. Esta reducción continuó con las sesiones de auto-exposición y se mantuvo a $0 \mathrm{du}$ rante todo el seguimiento.

En los seguimientos posteriores no se efectuó ningún registro formal, pero consideramos como válida la información del paciente a través de sus respuestas a una entrevista habitual en nuestro trabajo en la que confirmaba el mantenimiento de la mejoría. Si se mantuvieron los registros de pensamientos hipocondriacos, que no revelan ninguna alteración.

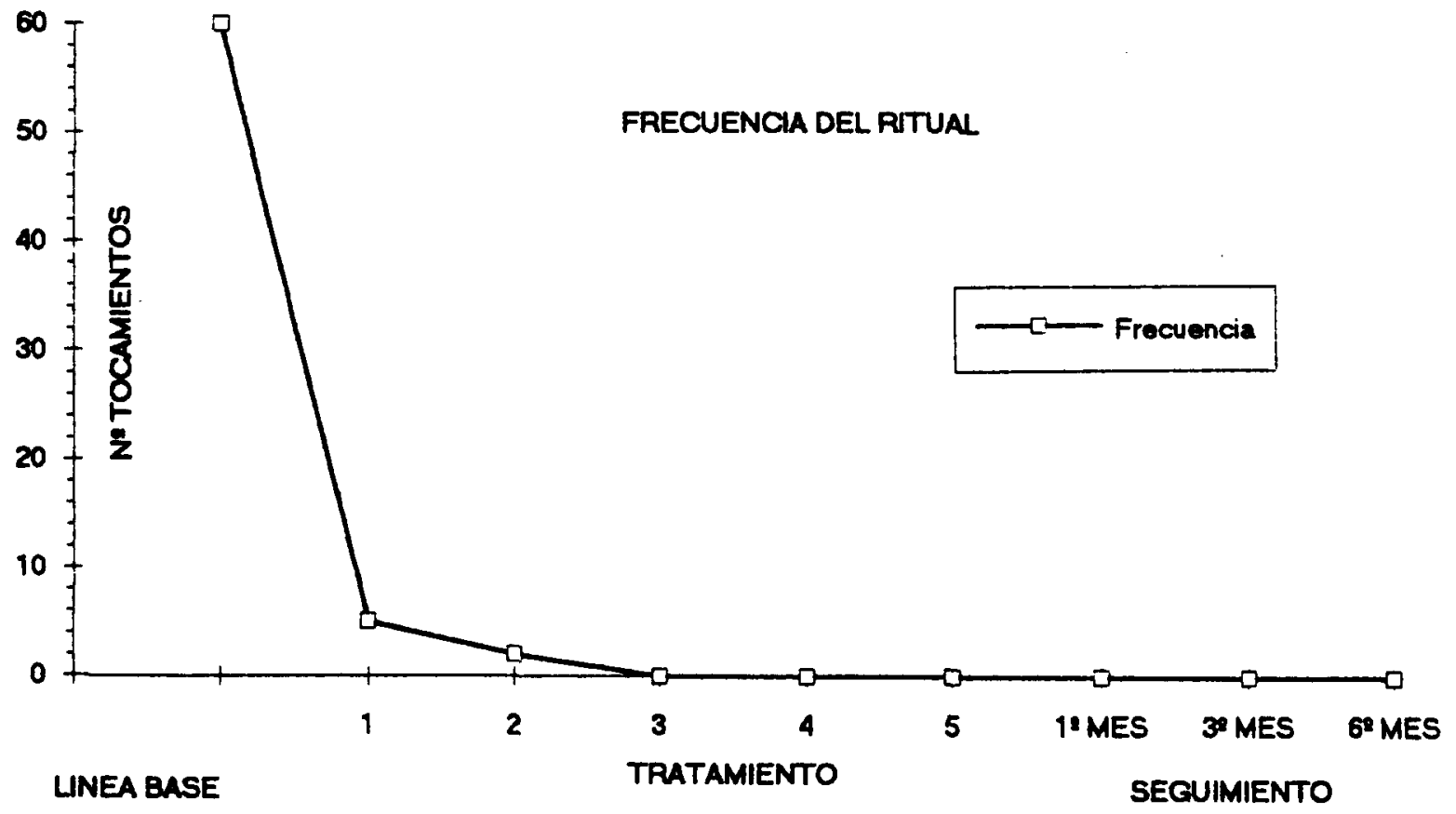

Figura 2.- 
En el seguimiento telefónico que se llevó a cabo apenas hace unos dias, $\mathrm{C}$ nos confirmó que continuaba perfectamente.

\section{4.- DISCUSIÓN}

Como mencionamos en la introducción, la hipocondria es, en ocasiones, difícil de distinguir clinicamente del trastorno obsesivocompulsivo. Estas ocasiones son, a nuestro juicio, aquellas en las que la conducta de enfermedad adquiere una forma particularmente ritualistica. El caso que hemos presentado es un ejemplo de lo anterior. Nuestra paciente mostraba una conducta ritual, la auto-exploración, como respuesta a una obsesión, tener un cáncer. Visto así, el diagnóstico más probable habría de ser el de TOC. Sin embargo, C presentaba también otros componentes tipicos del trastorno hipocondriaco, además de la preocupación por tener una enfermedad grave, como la reaparición de estas preocupaciones a pesar de las explicaciones médicas, la interpretación de signos o sintomas corporales como una señal del padecimiento, el temor a la enfermedad, y las conductas propias de un enfermo, como visitar al médico o explorarse con fines autodiagnósticos.

De otro lado, según el DSM IV (APA, 1994), sólo debe realizarse un diagnóstico de TOC si las obsesiones o compulsiones no se restringen a preocupaciones por una enfermedad. En este caso, aunque existian otros comportamientos obsesivos, como rituales de comprobación leves, estos no eran lo suficientemente graves como para justificar este diagnóstico.

Desde el ángulo inverso, en el TOC se dan con frecuencia conductas tipicas de enfermedad. Recientemente, Savron, Fava, Grandi, Rafanelli, Raffi, y Belluardo (1996), analizan en un estudio las relaciones entre el TOC y la hipocondria mediante la administración del EAE a pacientes obsesivo-compulsivos y controles normales. Los resultados indican una conducta de enfermedad levemente alterada en los pacientes obsesivos, que obtuvieron mayores puntuaciones en las escalas, pero con débil correlación entre los miedos y las creencias hipocondriacas y la temática de los sintomas obsesivo-compulsivos.

En cuanto al tratamiento seguido en el presente caso, se llevó a cabo partiendo del aná- lisis funcional, enriquecido éste con las aportaciones del modelo explicativo cognitivo, se consiguieron los objetivos previstos, y en el seguimiento, se apreció una clara estabilidad de la mejoria. Esos cambios los atribuimos a la intervención.

Al margen del diagnóstico psiquiátrico, desde la óptica de la terapia cognitivo-conductual, la estrecha relación entre evaluación y tratamiento permite no sólo identificar las variables dependientes, sino disenar un plan de intervención individual para cada paciente y evaluarlo de forma continuada lo cual, además, nos permite revisar y modificar nuestras hipótesis y/o revisar y modificar las estrategias de intervención con la finalidad de adaptarlas a nuestro paciente, a su problema concreto, a sus circunstancias personales, a su ambiente particular y al especial desarrollo de la intervención. En el tratamiento de $\mathrm{C}$, podemos observar cómo se intentan cumplir estas máximas:

1.- Se diseño el programa de intervención (con la colaboración de la propia paciente) escogiendo las técnicas que consideramos más eficaces para modificar las conductas anómalas que fueron perfilándose a través del análisis de la conducta de C. En concreto, basamos nuestro trabajo en una técnica sobre cuya eficacia hay pocas dudas: la exposición con prevención de respuesta.

2.- Se utilizaron medidas continuas, como el registro de conductas (autorregistro en este caso) que permiten observar los cambios que acontecen, tanto en las variables dependientes, como en otras circunstancias del sujeto que puedan interactuar con ellas.

3.- Se modificó el programa atendiendo a las especiales dificultades que iban surgiendo. Por ejemplo, se añadió una técnica, el habit reversal, para facilitar el cumplimiento de la prevención de respuesta, cuando observamos que ésta resultaba problemática.

Nótese que el número de sesiones fue minimo ( 7 sesiones de 30 minutos), se utilizaron técnicas especificas (la exposición con prevención de respuesta) y el resultado fue satisfactorio en un caso, la hipocondria, de considerable gravedad y repercusión individual y socio-sanitaria. En el contexto de un servicio público de salud mental resulta alentador pensar que un trastomo como éste pueda ser abordado con cierta eficacia, atendiendo a la especial situa- 
ción de presión asistencial, con una intervención corta y relativamente sencilla.

Para finalizar, al margen de estas últimas consideraciones, el caso que hemos descrito nos hace pensar que, en los pacientes hipocondriacos en los que el componente fundamental es el conductual, las técnicas de exposición, dentro de un programa más amplio que contemple el mayor número de variables problema del sujeto, son las más adecuadas, y que contribuyen indirectamente a propiciar los cambios cognitivos necesarios relacionados con la percepción de enfermedad, sin necesidad de utilizar estrategias cognitivas más complejas y elaboradas, que deberiamos reservar para los casos en que el trastorno pueda estar más relacionado con una alteración perceptivoatencional.

\section{REFERENCIAS BIBLIOGRAFICAS}

American Psychiatrlc Association (1994). Diagnostic and Statistical Manual of Mental Disorders (4" edición) (DSM IV). Washington, DC: APA.

Avia, M.D. (1993). Hipocondria. Barcelona: Martinez Roca.

Barsky, A.J., Cleary, P.D., Wyshak, G., Spitzer, R., Williams, J. y Klerman, G. (1992). A structured diagnostic interview for hypochondriasis: a proposed criterion standard. Joumal of Nervous and Mental Heatth, 180, 20-27.

Beck. A. T., Rush, J., Shaw, B. y Emery, G. (1983). Terapia Cognitiva de la Depresión. Bllbao: DDB.

Beck, A.T. (1988). Cognitive appproaches to panic disorders: theory and therapy. En S. Rachman y J.D. Maser (eds), Panic: psyhcological perspectives. Hillsdale, Nueva Jersey, L.E.A.

Bellack, A.S., y Hersen, M.H. (1977). Behaviour Modification: an introductory textbook. The Williams and Wilkins Co.

Bernstein D.A. y Borkovec T.D. (1973). Progressive Relaxation Training. Research Press.

Clark, D.M. (1988). A cognitive model of panic attacks. En Rachman, S., y Maser, J.D. (eds.), Panic: Psychological Perspectives. Erlbaum Hillsdale.

Easterning, D. y Leventhal, H. (1989). The contribution of concrete cognition to emotion: neutral symptoms as elicitors of worry about cancer. Joumal of Applied Psychology, 74, 787796.

Fava, G., Grandi, S., Michelacci, L., Saviotti, F., Conti, S., Bovicelli, L., Trombini, G., y Orlandi, C. (1990). Hipocondriacal fears and beliefs in pregnacy. Acta Psychiatrica Scandinavica, 82, 70-72.

Hitchcock, P.B., y Mathews, A. (1992). Interpretation of bodily symptoms in hypocondriasis. Behavior Researh and Therapy, 30 (3), 223-234.

Kellner, R. (1986). Somatization and hypochondriasis. Nueva York: Praeger.

Martinez y Botella (1996). Evaluación y tratamiento psicológico de la hipocondria: revisión y análisis critico. Psicologia Conductual. 4, 1, 29-62.

Salkovskis, P.M., y Clark, D.M. (1993). Panic disorder and hypochondriasis. Advances in Behaviour Research and Therapy, 15, 2348.

Savron, G., Fava, G.A., Grandi, S., Rafanelli, C, Raffi, A.R., y Belluardo, P (1996). Fears and hypochodriacal beliefs in obssesive-compulsive disorder. Acta Psychiatrica Scandinavica 93 (5): 345-348.

Wanwick, H., y Salkovskis, P. (1990). Hypocondriasis. Behavior Research and Therapy, 28, 105-117. 